\title{
Direct observation of the collapse of the delocalized excess electron in water
}

\author{
Savolainen, Janne ; Uhlig, Frank ; Ahmed, Saima ; Hamm, Peter ; Jungwirth, Pavel
}

\begin{abstract}
It is generally assumed that the hydrated electron occupies a quasi-spherical cavity surrounded by only a few water molecules in its equilibrated state. However, in the very moment of its generation, before water has had time to respond to the extra charge, it is expected to be significantly larger in size. According to a particle-in-a-box picture, the frequency of its absorption spectrum is a sensitive measure of the initial size of the electronic wavefunction. Here, using transient terahertz spectroscopy, we show that the excess electron initially absorbs in the far-infrared at a frequency for which accompanying ab initio molecular dynamics simulations estimate an initial delocalization length of $40 \AA$. The electron subsequently shrinks due to solvation and thereby leaves the terahertz observation window very quickly, within $200 \mathrm{fs}$.
\end{abstract}

DOI: https://doi.org/10.1038/nchem.1995

Posted at the Zurich Open Repository and Archive, University of Zurich

ZORA URL: https://doi.org/10.5167/uzh-99212

Journal Article

Accepted Version

Originally published at:

Savolainen, Janne; Uhlig, Frank; Ahmed, Saima; Hamm, Peter; Jungwirth, Pavel (2014). Direct observation of the collapse of the delocalized excess electron in water. Nature Chemistry, 6(8):697-701.

DOI: https://doi.org/10.1038/nchem.1995 


\title{
Direct Observation of the Collapse of the Delocalized Excess Electron in Water
}

\author{
Janne Savolainen ${ }^{1 *}$, Frank Uhlig ${ }^{2 *}$, Saima Ahmed ${ }^{1}$, Peter Hamm ${ }^{1 \#}$, Pavel Jungwirth ${ }^{2 \#}$ \\ ${ }^{1}$ Department of Chemistry, University of Zurich, \\ Winterthurerstrasse 190, CH-8057 Zurich, Switzerland \\ ${ }^{2}$ Institute of Organic Chemistry and Biochemistry, \\ Academy of Sciences of the Czech Republic, \\ Flemingovo nam. 2, 16610 Prague 6, Czech Republic \\ ${ }^{*}$ Both authors contributed equally \\ \# corresponding authors: phamm@pci.uzh.ch, \\ pavel.jungwirth@marge.uochb.cas.cz
}

(Dated: July 8, 2014)

\begin{abstract}
It is generally assumed that the hydrated electron occupies a quasi-spherical cavity surrounded by only a few water molecules in its equilibrated state. However, in the very moment of its generation before water had time to respond to the extra charge, it is expected to be significantly larger in size. According to a particle-in-a-box picture, the frequency of its absorption spectrum is a sensitive measure of the initial size of the electronic wave function. Here, we show with the help of transient terahertz spectroscopy that it initially absorbs in the far-IR at a frequency for which accompanying $a b$ initio molecular dynamics simulations estimate an initial delocalization length of $\approx 40 \AA$. The electron subsequently shrinks due to solvation and thereby leaves the $\mathrm{THz}$ observation window very quickly within $\approx 200 \mathrm{fs}$.
\end{abstract}

One of the simplest transient species, the hydrated, or aqueous electron $\left(\mathrm{e}_{\mathrm{aq}}^{-}\right)$, is a highly reactive intermediate and as such an important entity for example in radiation chemistry $[1,2]$. But, perhaps even more compellingly, $e_{a q}^{-}$is the simplest case of a quantum particle in a complex chemical and thermal environment; an object of great fundamental interest that has challenged researchers since it's discovery $[3,4]$. To appreciate the system in full, it is vital to understand the initial steps from the photodetachment to the $\mathrm{e}_{\mathrm{aq}}^{-}$formation. Femtosecond pump-probe experiments have been used to unravel details of the equilibration processes following the trapping by the solvent [5-12]. In addition, picosecond measurements on the recombination kinetics of $\mathrm{e}_{\mathrm{aq}}^{-}$have described the ejected electron's migration lengths from its geminate partners [13-22]. These studies evoke a picture of an excess electron that is at first delocalized in the conduction band and then localizes to the hydrated electron $\mathrm{e}_{\mathrm{aq}}^{-}$within about a picosecond. According to a simple particle-in-a-box picture, such a localization should be accompanied by a shift in the absorption spectrum from potentially the $\mathrm{THz}$ or even $\mathrm{GHz}$ regions over the mid-IR to the VIS spectral range. The latter stages of this process have indeed been observed by femtosecond pump-probe experiments in the mid-IR [9-11, 23, 24] or NIR/VIS regime $[10,14,24]$, but the initial frequency at which this process starts, which is directly related to the initial degree of delocalization of the excess electron, has not yet been explored.

According to the current knowledge, there exists a threshold in the photodetachment between molecularbased mechanisms and bulk excitation to delocalized states [17, 21]. With high enough energies given to the ejected electron, the conduction band can be directly reached, leaving the nuclear positions unchanged. Given the inhomogeneity in the water hydrogen bond- ing network, the threshold energy for this vertical ionization mechanism is $\approx 9.8-9.9 \mathrm{eV}$ [21], about $0.5-1 \mathrm{eV}$ below the vacuum level (it should be added however that other works have estimated a much smaller width of the conduction band based on an extrapolation from clusters [25]). Below this threshold, the conduction band is not directly accessible and $e_{a q}$ is formed via mechanisms involving nuclear degrees of freedom in the sense of solvation, or directly of the excited water molecule.

The $\mathrm{e}_{\mathrm{aq}}^{-}$'s electronic structure is understood to be $s$ like in character with the absorption relating primarily to the $\mathrm{s} \rightarrow \mathrm{p}$ transition. Rossky and coworkers have pioneered this picture based on pseudopotential calculations [26], revealing a $\mathrm{e}_{\mathrm{aq}}^{-}$that occupies a quasi-spherical cavity with a radius of about $2.5 \AA$ surrounded by $4-6$ water molecules. Recent $a b$ initio molecular dynamics simulations show that $\mathrm{e}_{\mathrm{aq}}^{-}$has a rather complex structure. Its largest part resides in a cavity, however, there is also a sizeable diffuse part, as well as a significant part that overlaps with the surrounding water molecules. [27] This picture is somewhat in between the consensus based on most of the pseudopotential calculations [26, 28] and a rivaling new pseudopotential model, predicting a significantly larger size of a strongly delocalized hydrated electron even in the equilibrated ground state. [29, 30] Note that an ab initio molecular dynamics approach by construct accounts for more quantum electronic structure effects than pseudopotentials, with the latter being also more prone to potential parametrization artifacts.

The $\mathrm{THz}$ response of a solvated electron has been exploited before, however only in $n$-hexane, in which solvation is so weak that the electron remains delocalized for very long time [31]. Here, we extend the concept to water. That is, we present direct evidence of a delocalized electron right after photoionization using optical-pumpTHz-probe spectroscopy, which is very short-lived, and 


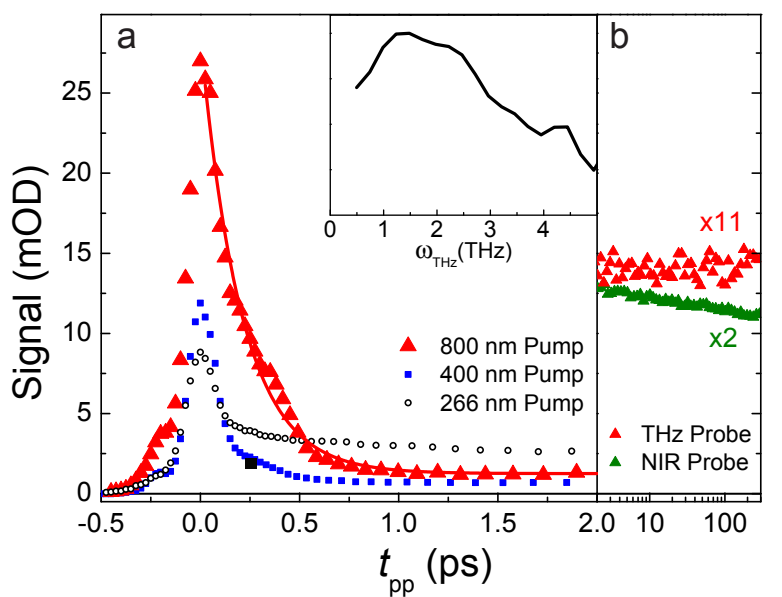

FIG. 1: The THz response to photoionization of neat water. (a) $800 \mathrm{~nm}$ pump (red triangles) with a single exponential fit (200 fs, red line), $400 \mathrm{~nm}$ pump (blue squares) and $266 \mathrm{~nm}$ pump (black circles). The 400 and $266 \mathrm{~nm}$ pump traces are scaled to the $e_{\mathrm{aq}}^{-}$concentration in the $800 \mathrm{~nm}$ pump measurement. inset: Spectral signature of the excess electron upon $800 \mathrm{~nm}$ pumping deduced from a 2D scan. This initial spectrum peaks at about $1.5 \mathrm{THz}$. (b) The long time THz response with $800 \mathrm{~nm}$ pump (red, scaled by factor of 11) stays constant whereas the response probed at NIR shows clearly the geminate recombination kinetics (green).

follow its localization upon solvation. Corresponding $a b$ initio molecular dynamics simulations capture the subsequent stages of electron localization, bridging thus the domains of the present time-resolved $\mathrm{THz}$ measurement with the previous ultrafast experiments in the mid-IR to VIS spectral regimes [9-11, 14, 23, 24].

\section{RESULTS}

\section{A. Experimental}

Fig. 1 shows the $\mathrm{THz}$ response of the excess electron in water as a function of the pump-probe delay time $t_{\mathrm{pp}}$, with the $\mathrm{THz}$ scanning time $t_{\mathrm{THz}}$ kept fixed to the peak of the $\mathrm{THz}$ probe field. Pump pulses at $266 \mathrm{~nm}, 400 \mathrm{~nm}$ and $800 \mathrm{~nm}$ were used with irradiances of $0.08 \mathrm{TW} / \mathrm{cm}^{2}, 0.3 \mathrm{TW} / \mathrm{cm}^{2}$ and $1.9 \mathrm{TW} / \mathrm{cm}^{2}$, respectively, which resulted in $\mathrm{e}_{\mathrm{aq}}^{-}$concentrations of $20 \mu \mathrm{M}$, $110 \mu \mathrm{M}$ and $65 \mu \mathrm{M}$, as determined from accompanying experiments using a 800 -nm probe pulse (Supplementary Figure 4) [32]. While it is well established that $266 \mathrm{~nm}$ and $400 \mathrm{~nm}$ pumping generates $\mathrm{e}_{\mathrm{aq}}^{-}$by multiphoton processes, photolysis of neat water with $800 \mathrm{~nm}(1.54 \mathrm{eV})$ pulses has been explored much less [33, 34], because the ionization process is not nearly as well characterized in this case. The matching irradiance dependencies of $\mathrm{THz}$ and NIR responses shown in Supplementary Figure 2 prove that the $\mathrm{THz}$ signal indeed originates from excess electrons for all pump-wavelengths. The $266 \mathrm{~nm}$ and
$400 \mathrm{~nm}$ pump data follow the expected second- and thirdorder power laws [24,35], whereas the $800 \mathrm{~nm}$ pump signal depicts a threshold behavior with $\approx 1 \mathrm{TW} / \mathrm{cm}^{2}$ as the onset of ionization. Such a threshold behavior indicates that a strong-field ionization rather than a multiphoton mechanism is behind the photo detachment with $800 \mathrm{~nm}$ photons, in accordance with the conclusions from Ref. [34]. Also the signal drop with circularly polarized pump light shown in Supplementary Figure 3 provides strong evidence for that interpretation [36].

Geminate recombination of $\mathrm{e}_{\mathrm{aq}}^{-}$proceeds in a diffusive manner and hence is a measure of the initial delocalization length, which in turn has been shown to depend on the excitation energy [14, 18-21, 37, 38]. Supplementary Figure 4 shows the recombination kinetics measured for 266-nm, 400-nm and 800-nm pumping, respectively. Comparing the recombination kinetics with Refs. [20, 21], we estimate that $800-\mathrm{nm}$ pumping result in an effective excitation energy of $12.4 \mathrm{eV}$ and an average ejection length of $\approx 38 \AA$. At this energy the electron reaches the conduction band vertically [19], or even states above the vacuum level (which we expect to relax very quickly in bulk solution).

With that in mind, we return to the discussion of the $\mathrm{THz}$ response (Fig. 1). During the first few hundred femtoseconds, the $\mathrm{THz}$ responses for all pump-wavelengths exhibit a fast rise to a peak value and a subsequent decay (Fig. 1a). The $800 \mathrm{~nm}$ pump trace has the largest amplitude and an initial decay with a time constant of $\approx 0.2 \mathrm{ps}$ down to a small offset that remains constant until the end of the scanning range (Fig. 1b, red). The asymmetric shape of the initial signal indicates that the decay is not instrument response limited. The $266 \mathrm{~nm}$ pump trace, in contrast, initially exhibits an much sharper, symmetric spike, which we consider to represent the instrument response. The long term offset decays gradually afterwards. The $400 \mathrm{~nm}$ pump trace depicts an intermediate behavior having some sub-ps decay after the instrument response limited peak. With respect to the initial evolution of the ejected electron, the $800 \mathrm{~nm}$ pump data is the most conclusive and we will focus our discussion on it.

The inset of Fig. 1a shows the spectral signature of the water response upon $800 \mathrm{~nm}$ pumping, which has been extracted from a full 2D data set along both the pumpprobe delay time $t_{\mathrm{pp}}$ and $\mathrm{THz}$ scanning time $t_{\mathrm{THz}}$ (see Supplementary Figure 5 and Supplementary Information for details) [39, 40]. The spectral response shows a broad band peaking at $\approx 1.5 \mathrm{THz}$ with a long tail towards larger frequencies. In essence, the band shown in the inset of Fig. 1a decays according to the red line of Fig. 1a, while the sensitivity of the method to details of the data does not allow us to extract an additional time evolution of the spectral response as it decays.

The $\mathrm{THz}$ response could either be of electronic nature or due to a change in the nuclear spectrum of a perturbed water network. For example, the change in the molar extinction coefficient of water induced by dissolving $\mathrm{NaCl}$ is $\approx 10^{-2} \mathrm{mM}^{-1} \mathrm{~cm}^{-1}$ [41]. Assuming that $\mathrm{e}_{\mathrm{aq}}^{-}$and the 
geminate partners have a similar effect on the $\mathrm{THz}$ water spectrum, the induced absorption change would be as small as $<0.05 \mathrm{mOD}$ at the concentration of $\mathrm{e}_{\mathrm{aq}}^{-}$generated in our experiment; several orders of magnitude smaller than the detected signal. The temperature jump induced by the pump-process also disturbs the water network to a certain extent, but we estimate a temperature jump of $\approx 0.2{ }^{\circ} \mathrm{C}$, which in turn would result in an increase of $\mathrm{THz}$ water absorption of about $\approx 0.4 \mathrm{mOD}$ [42], again not sufficient to explain the detected signal peaking at about $27 \mathrm{mOD}$.

On the other hand, the $\approx 1 \mathrm{mOD}$ signal size of the long-time offset (Fig. 1b, red) is in fair agreement with our estimate of a temperature effect. Indeed, that signal stays constant in time up to $300 \mathrm{ps}$, as expected for a temperature jump, whereas the NIR-probe signal (Fig. 1b, green), which probes geminate recombination of $e_{\text {aq }}^{-}$, does decay by a bit. It should however be mentioned that a long-time signal is also observed for $266-\mathrm{nm}$ and 400-nm pumping, which is larger and not constant in time but rather parallels geminate recombination, so it cannot be exclusively due to heating. The effect is currently not understood and likely is related to the smaller ejection length and different detachment mechanisms at these pump wavelengths.

Nonetheless, the large amplitude of the initial THz signal upon 800-nm pumping can only be explained as an electronic response. The signal size of the initial peak for $\mathrm{THz}$ probing and that observed after equilibration in the NIR regime are of similar magnitudes (i.e., $\approx 27 \mathrm{mOD}$ and $7 \mathrm{mOD}$, respectively, see Fig. 1a and Supplementary Figure 4). This equivalence is expected for a particle-ina-box description with an oscillator strength $f \propto \nu|\mu|^{2}$, whose transition frequency $\nu$ scales as $1 / r_{\mathrm{g}}^{2}$ (where $r_{\mathrm{g}}$ is the dimension of the box) and the transition dipole $\mu$ as $r_{\mathrm{g}}$. Hence, as the initially delocalized electron localizes, its frequency and thus its size varies over a wide range (by factors 300 or 0.06 , respectively), while its oscillator strength stays constant (within a factor 4). Another interesting aspect of the $\mathrm{THz}$ response is the complete lack of anisotropy (see Supplementary Figure 3) indicating that the ejected electron has spherical symmetry from the very beginning as expected for a $s$-like wavefunction.

\section{B. Computational}

In order to aid the interpretation of the experiment and to put it onto more quantitative grounds, we modeled the localization process with ab initio molecular dynamics (AIMD) simulations. An averaged time evolution of $r_{\mathrm{g}}$ and the lowest excitation energy are shown in Fig. 2 together with the mean deviations. An exponential fit of the averaged $r_{\mathrm{g}}$ reveals a time constant of $1 \mathrm{ps}$. At the moment of electron attachment to the neutral water system, the excitation energies for the excess electron are strongly red-shifted compared to the optical absorption of a hydrated electron in equilibrium. However, as the ex-

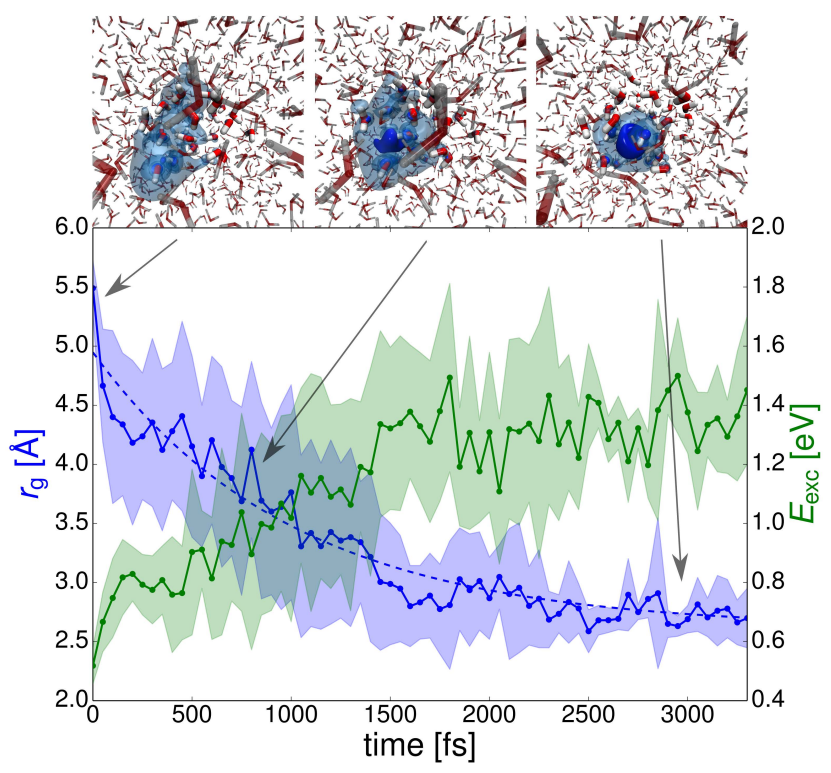

FIG. 2: Averaged radius of gyration $r_{\mathrm{g}}$ (blue) and lowest excitation energy (green) of an excess electron attached to neat water system as a function of time. Data are calculated every $50 \mathrm{fs}$ using TD-DFT and are presented together with shading depicting the corresponding mean deviations and an exponential fit with a $1 \mathrm{ps}$ time constant (dashed line). Three exemplary snapshots along a representative trajectory are presented on the top of the graph with isosurfaces of the spin density shown in blue (transparent at 0.0001 and opaque at $0.001 \mathrm{Bohr}^{-3}$ ). The QM water molecules are shown in an opaque licorice representation and MM water molecules in a transparent licorice representation.

cess electron localizes and shrinks, the optical spectrum converges to that of a hydrated electron.

The maximum size of the attached electron in our simulations is limited by the size of the QM subsystem to roughly $7 \AA$. The simulations thus aim at filling the gap between the initially strongly delocalized electron absorbing in the $\mathrm{THz}$ region and the (practically) localized electron probed in the NIR spectral region. In order to get even closer to the experimentally measured absorption in the $\mathrm{THz}$ region, additional electron attachment calculations to neat water systems with larger QM subsystems (either $64 \mathrm{QM}$ and $960 \mathrm{MM} \mathrm{H}_{2} \mathrm{O}$, or $256 \mathrm{QM}$ and $3886 \mathrm{QM} \mathrm{H}_{2} \mathrm{O}$ ) have been performed. In this case, we recorded only the initial size $(t=0)$ of an excess electron attached to snapshots sampled from 6 trajectories of neat water covering almost $30 \mathrm{ps}$, yielding electron sizes in the range of 6 - $10 \AA$.

Fig. 3 depicts the first excitation energy plotted against the $r_{\mathrm{g}}$ of the excess electron from all the above simulations. The dependence reminds of the behavior of a particle-in-a-box system. We have, however, shown previously that the hydrated electron is of a much more complex nature. [27] Nevertheless, it is still possible to fit the data accurately using a particle-in-a-box formula, i.e., the $a / r_{g}^{2}$ relationship, which indicates that this behavior of the hydrated electron is rather robust with re- 


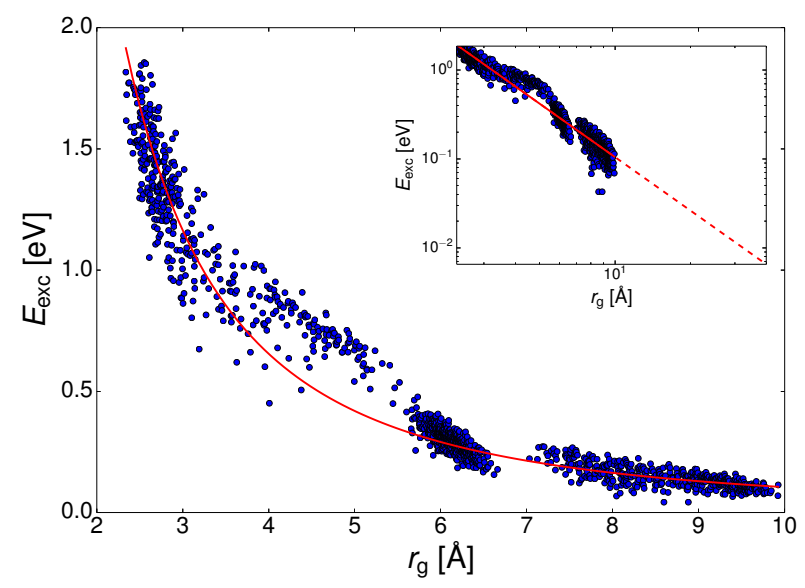

FIG. 3: Correlation between radius of gyration $r_{\mathrm{g}}$ of the spin density and first excitation energy (blue dots). Red line shows fit of data to $a / r_{\mathrm{g}}^{2}$ with $a=10.5 \mathrm{eV}^{2}$ (covariance $\sigma=2$. $10^{-3} \mathrm{eV} \AA^{2}$ ). This fit is very close to that using parameters for a 3D particle-in-a-box with the mass of an electron (see Supplementary Information, Sec. II.B and Supplementary Figure 6) Inset depicts same data with extended $r_{\mathrm{g}}$ axis and correspondingly extended fit curve (dashed).

spect to the particular model employed for its description [28]. The present fitting allows us to extrapolate to size and excitation energy ranges which are not directly accessible to the AIMD simulations. Although the simulated data exhibit a certain spread, they can clearly be fitted to a straight line in the log-log plot and faithfully extended to electron sizes pertinent to the $\mathrm{THz}$ region. From such an extrapolation, the radius of gyration of an excess electron with a first excitation energy of $1.5 \mathrm{THz}$, as determined experimentally (Fig. 1a, inset), would be about $40 \AA$ (see fit in Fig. 3). It is remarkable that this number is very close to the ejection length, which has independently been estimated from the recombination kinetics (see Supplementary Figure 4) along the lines of Ref. [21].

\section{DISCUSSION}

The picture that emerges from our combined experimental-theoretical study is schematically depicted in Fig. 4. The initially generated electron in the present experiment is rather delocalized with a radius of at least $r_{g}=40 \AA$ (we cannot entirely exclude that electron delocalization is even larger at the very moment of the photoionization event). It is reasonable to assume that the initially generated electron is essentially centered around the ionized water molecule (Fig 4a). The water molecules then solvate the electron, and its size shrinks considerably. If the probability to get trapped at a particular position is proportional to the initial electron density, then the mean distance of the localized electron from the origin roughly equals to its original radius of gyration (Fig. 4b), explaining why initial delocalization length and (b)

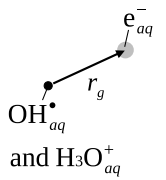

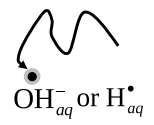

FIG. 4: A schematic depiction of the excess electron formation and localization process in water. (a) The ejected delocalized excess electron (in gray) with radius $r_{\mathrm{g}}$, (b) collapse of the electron wavefunction, which will result in an average ejection length $r_{\mathrm{g}}$, and (c) diffusive geminate recombination.

final ejection length are the same within error bars. During the complete collapse of the electron wave function occurring on a 1 ps timescale (Fig. 2), the absorption sweeps from the $\mathrm{THz}$ into NIR regime. That is, it leaves our $\mathrm{THz}$ observation window after $\approx 200 \mathrm{fs}$ (Fig. 1), then appears in the mid-IR [9-11, 23, 24] and eventually the NIR spectral range upon full solvation $[10,14,24]$. Afterwards, the hydrated electron recombines in a diffusive process on a much slower timescale (Fig. 4c).

In conclusion, time-resolved $\mathrm{THz}$ spectroscopy captures the early stages of the photodetached electron and furthermore provides a direct measure of its initial size. The ejection length after solvation agrees extremely well with the initial size of the electron estimated from the $\mathrm{THz}$ experiment together with the ab initio molecular dynamics simulations. To date, the only experimental measure of the initial size of the electron has used the geminate recombination kinetics of the $e_{a q}^{-} /$hole pair as an indirect 'ruler', the standing hypothesis being that the ejection length is directly related to the initial size of the electron's wavefunction [19]. However, seeing is believing, and we can now conclude that this assumption is indeed correct, at least in the case of above threshold photolysis. Thus, our experimental and computational studies paint a more complete picture of the initial steps involved in the $e_{\mathrm{aq}}^{-}$formation, with the electron resonance sweeping continuously through the spectrum from the $\mathrm{THz}$ regime into the NIR regime as a the electron localizes. The work paves the way towards understanding water's character as a temporary liquid semiconductor.

\section{METHODS}

The response of the excess electron in water was measured using a optical-pump THz-probe setup [43] with the spectrum of the $\mathrm{THz}$ pulses peaking at $\approx 1.0 \mathrm{THz}$ and covering a bandwidth of approximately $0.3-5 \mathrm{THz}$. For the AIMD simulations, an electron is vertically attached into the conduction band (which is $0.5-1 \mathrm{eV}$ below the vacuum level according to our AIMD model) to a neat water system of 32 quantum mechanical (QM) 
water molecules surrounded by 992 molecular mechanical (MM) water molecules with periodic boundary conditions. Data were collected from seven independent trajectories. The optical spectrum was calculated along the trajectories every $50 \mathrm{fs}$. The size of the excess electron during the localization was taken as the radius of gyration of the spin density (see Supplementary Information and Supplementary Figure 1 for details).

\section{Acknowledgments}

We thank Steven Bradforth and Petr Kužel for valuable discussions. The work has been supported in part by the Swiss National Science Foundation (SNF) through the NCCR MUST. PJ acknowledges the Czech Science Foundation (Grant P208/12/G016) for support and thanks the Academy of Sciences for the Praemium Academie award. FU and PJ also gratefully acknowledge the computing time granted by the John von Neumann Institute for Computing (NIC) and provided on the supercomputer JUROPA at Jülich Supercomputing Centre (JSC). Correspondence and requests for materials should be addressed to Peter Hamm (experiment) and Pavel Jungwirth (calculations).
[1] Garrett, B. C. et al. Role of water in electron-initiated processes and radical chemistry: Issues and scientific advances. Chem. Rev. 105, 355-389 (2005).

[2] Young, R. M. \& Neumark, D. M. Dynamics of solvated electrons in clusters. Chem. Rev. 112, 5553-5577 (2012).

[3] Hart, E. J. \& Boag, J. W. Absorption spectrum of hydrated electron in water and in aqueous solutions. J. Am. Chem. Soc. 84, 4090-4095 (1962).

[4] Boag, J. W. \& Hart, E. J. Absorption spectra in irradiated water and some solutions - absorption spectra of hydrated electron. Nature 197, 45-47 (1963).

[5] Chase, W. J. \& Hunt, J. W. Solvation time of electron in polar liquids - water and alcohols. J. Phys. Chem. 79, 2835-2845 (1975).

[6] Wiesenfeld, J. M. \& Ippen, E. P. Dynamics of electron solvation in liquid water. Chem. Phys. Lett. 73, 47-50 (1980).

[7] Migus, A., Gauduel, Y., Martin, J. L. \& Antonetti, A. Excess electrons in liquid water - 1st evidence of a prehydrated state with femtosecond lifetime. Phys. Rev. Lett. 58, 1559-1562 (1987).

[8] McGowen, J. L., Ajo, H. M., Zhang, J. Z. \& Schwartz, B. J. Femtosecond studies of hydrated electron recombination following multiphoton ionization at 390-nm. Chem. Phys. Lett. 231, 504-510 (1994).

[9] Pepin, C., Goulet, T., Houde, D. \& Jay-Gerin, J. P. Observation of a continuous spectral shift in the solvation kinetics of electrons in neat liquid deuterated water. $J$. Phys. Chem. A 101, 4351-4360 (1997).

[10] Laenen, R., Roth, T. \& Laubereau, A. Novel precursors of solvated electrons in water: Evidence for a charge transfer process. Phys. Rev. Lett. 85, 50-53 (2000).

[11] Vilchiz, V. H., Kloepfer, J. A., Germaine, A. C., Lenchenkov, V. A. \& Bradforth, S. E. Map for the relaxation dynamics of hot photoelectrons injected into liquid water via anion threshold photodetachment and above threshold solvent ionization. J. Phys. Chem. A $\mathbf{1 0 5}$, 1711-1723 (2001).

[12] Laenen, R. \& Roth, T. Generation of solvated electrons in neat water: new results from femtosecond spectroscopy. J. Mol. Struct. 598, 37-43 (2001).

[13] Crowell, R. A. \& Bartels, D. M. Multiphoton ionization of liquid water with 3.0-5.0 eV photons. J. Phys. Chem. 100, 17940-17949 (1996).
[14] Thomsen, C. L., Madsen, D., Keiding, S. R., Thogersen, J. \& Christiansen, O. Two-photon dissociation and ionization of liquid water studied by femtosecond transient absorption spectroscopy. J. Chem. Phys. 110, 3453-3462 (1999).

[15] Bartels, D. M. \& Crowell, R. A. Photoionization yield vs energy in h2o and d2o. J. Phys. Chem. A 104, 3349-3355 (2000).

[16] Sander, M. U., Gudiksen, M. S., Luther, K. \& Troe, J. Liquid water ionization: mechanistic implications of the $\mathrm{h} / \mathrm{d}$ isotope effect in the geminate recombination of hydrated electrons. Chem. Phys. 258, 257-265 (2000).

[17] Son, D. H., Kambhampati, P., Kee, T. W. \& Barbara, P. F. Delocalizing electrons in water with light. J. Phys. Chem. A 105, 8269-8272 (2001).

[18] Kee, T. W., Son, D. H., Kambhampati, P. \& Barbara, P. F. A unified electron transfer model for the different precursors and excited states of the hydrated electron. $J$. Phys. Chem. A 105, 8434-8439 (2001).

[19] Kambhampati, P., Son, D. H., Kee, T. W. \& Barbara, P. F. Solvation dynamics of the hydrated electron depends on its initial degree of electron delocalization. $J$. Phys. Chem. A 106, 2374-2378 (2002).

[20] Lian, R., Oulianov, D. A., Shkrob, I. A. \& Crowell, R. A. Geminate recombination of electrons generated by abovethe-gap $(12.4 \mathrm{eV})$ photoionization of liquid water. Chem. Phys. Lett. 398, 102-106 (2004).

[21] Elles, C. G., Jailaubekov, A. E., Crowell, R. A. \& Bradforth, S. E. Excitation-energy dependence of the mechanism for two-photon ionization of liquid $\mathrm{H}_{2} \mathrm{O}$ and $\mathrm{D}_{2} \mathrm{O}$ from 8.3 to $12.4 \mathrm{eV}$. J. Chem. Phys. 125, 044515 (2006).

[22] Kratz, S., Torres-Alacan, J., Urbanek, J., Lindner, J. \& Vöhringer, P. Geminate recombination of hydrated electrons in liquid-to-supercritical water studied by ultrafast time-resolved spectroscopy. Phys. Chem. Chem. Phys. 12, 12169-12176 (2010).

[23] Hertwig, A., Hippler, H. \& Unterreiner, A. N. Transient spectra, formation, and geminate recombination of solvated electrons in pure water UV-photolysis: an alternative view. Phys. Chem. Chem. Phys. 1, 5633-5642 (1999).

[24] Birkedal, V. et al. Observation of a persistent infrared absorption following two photon ionization of liquid water. Chem. Phys. 328, 119-124 (2006). 
[25] Coe, J. V. et al. Using cluster studies to approach the electronic structure of bulk water: Reassessing the vacuum level, conduction band edge, and band gap of water. J. Chem. Phys. 107, 6023-6031 (1997).

[26] Turi, L. \& Rossky, P. J. Theoretical studies of spectroscopy and dynamics of hydrated electrons. Chem. Rev. 112, 5641-5674 (2012)

[27] Uhlig, F., Marsalek, O. \& Jungwirth, P. Unraveling the complex nature of the hydrated electron. J. Phys. Chem. Lett. 3, 3071-3075 (2012).

[28] Jacobson, L. D. \& Herbert, J. M. Theoretical characterization of four distinct isomer types in hydratedelectron clusters, and proposed assignments for photoelectron spectra of water cluster anions. J. Am. Chem. Soc. 133, 19889-19899 (2011).

[29] Larsen, R. E., Glover, W. J. \& Schwartz, B. J. Does the hydrated electron occupy a cavity? Science 329, 65-69 (2010).

[30] Casey, J. R., Kahros, A. \& Schwartz, B. J. To be or not to be in a cavity: The hydrated electron dilemma. $J$. Phys. Chem. B 117, 14173-14182 (2013).

[31] Knoesel, E., Bonn, M., Shan, J. \& Heinz, T. F. Charge transport and carrier dynamics in liquids probed by $\mathrm{THz}$ time-domain spectroscopy. Phys. Rev. Lett. 86, 340-343 (2001).

[32] Hare, P. M., Price, E. A. \& Bartels, D. M. Hydrated electron extinction coefficient revisited. J. Phys. Chem. A 112, 6800-6802 (2008).

[33] Brown, M. S., Erickson, T., Frische, K. \& Roquemore, W. M. Hot electron dominated rapid transverse ionization growth in liquid water. Opt. Express 19, 12241 (2011).

[34] Li, J., Nie, Z., Zheng, Y. Y., Dong, S. \& Loh, Z.-H. Elementary electron and ion dynamics in ionized liquid water. J. Phys. Chem. Lett. 4, 3698-3703 (2013).

[35] Crowell, R. A. et al. Light-induced temperature jump causes power-dependent ultrafast kinetics of electrons generated in multiphoton ionization of liquid water. $J$. Phys. Chem. A 108, 9105-9114 (2004).

[36] Mics, Z. et al. Nonresonant ionization of oxygen molecules by femtosecond pulses: plasma dynamics studied by time-resolved terahertz spectroscopy. J. Chem. Phys. 123, 104310 (2005).

[37] Pimblott, S. M. Independent pairs modeling of the kinetics following the photoionization of liquid water. $J$. Phys. Chem. 95, 6946-6951 (1991).

[38] Goulet, T. \& Jay-Gerin, J. P. On the reactions of hydrated electrons with $\mathrm{OH}^{-}$and $\mathrm{H}_{3} \mathrm{O}^{+}$- analysis of photoionization experiments. J. Chem. Phys. 96, 5076-5087 (1992).

[39] Němec, H., Kadlec, K. \& Kužel, P. Methodology of an optical pump-terahertz probe experiment: An analytical frequency domain approach. J. Chem. Phys. 117, 8454 (2002).

[40] Kužel, P., Němec, H. \& Kadlec, K. Propagation of THz pulses in photoexcited media: Analytical theory for layered systems. J. Chem. Phys. 127, 024506 (2007).

[41] Xu, J., Plaxco, K. W., Allen, S. J., Bjarnason, J. E. \& Brown, E. R. 0.15-3.72 THz absorption of aqueous salts and saline solutions. Appl. Phys. Lett. 90, 031908 (2007).

[42] Zelsmann, H. R. Temperature-dependence of the opticalconstants for liquid $\mathrm{H}_{2} \mathrm{O}$ and $\mathrm{D}_{2} \mathrm{O}$ in the far IR region. J. Mol. Struct. 350, 95-114 (1995).

[43] Savolainen, J., Ahmed, S. \& Hamm, P. Two-dimensional Raman-terahertz spectroscopy of water. Proc. Natl. Acad. Sci. USA 110, 20402-20407 (2013). 